\title{
Assessment of dynamic balance during step initiation in Parkinson's disease patients and elderly - a validity study
}

\author{
GrZEGORZ JuRAs*, ANNA KAMIENIARZ, JUSTyNA MichalSKa, KAJETAN SŁOMKA \\ Institute of Sport Sciences, The Jerzy Kukuczka Academy of Physical Education in Katowice, Poland.
}

\begin{abstract}
Purpose: The aim of this study was to evaluate the reliability of the novel posturographic procedure and to investigate the relationships between parameters of this procedure and clinical tests. We hypothesised the proposed step initiation procedure to be reliable method of balance and motor disability assessment, especially in patients with neurological deficits. Also, we assumed high significant correlation between parameters of step initiation procedures and clinical tests. Methods: The 35 subjects with idiopathic PD and 35 agedmatched healthy controls participated in this study. The gait initiation was measured using two force platforms. The procedure consisted of three phases: (1) quiet standing on a first platform (2) crossing on the second platform, (3) quiet standing on a second platform. Testing was carried out in four conditions: unperturbed trial, obstacle crossing, step-up and step-down. Results: In the proposed gait initiation procedure, the $\mathrm{vCOP}$, raCOP and rmsCOP showed an excellent reliability $(I C C>0.80)$. For transit phase, reliability of all variables in all conditions also was excellent $(I C C=0.8-0.9)$. There were only a few associations between Tinetti scores and posturographic variables in controls, but in PD patients the significant correlations were found between the proposed measures and the UPDRS, Tinetti, FRT, TUG and with the BBS. Conclusions: The proposed gait initiation procedure is reliable and very suitable for the assessment of patients with Parkinson's disease. It can be used as an objective assessment of the clinical condition and dynamic balance, and help in the designing and programming of the appropriate rehabilitation and treatment.
\end{abstract}

Key words: Parkinson's disease, elderly, postural stability, validity, step initiation

\section{Introduction}

Human balance is maintained by a posture control mechanism which maintain the center of mass (COM) above the base of support. Three major sensory systems are involved in keeping the balance and upright posture: the vestibular, vision and somatosensory systems [31]. The body balance is affected when part of the control system is not working correctly, what may be caused by the ageing process [3]. In elderly, degeneration occurs in both sensory and musculoskeletal systems [25]. The function of sensory receptors deteriorates, what has a greater impact on the balance in elderly than in the young population [30]. Balance disorders do not only affect the elderly, but also can occur in patients with neurodegenerative diseases, for instance, the Parkinson's disease (PD). It is known that the decline in mobility and motor skills in PD patients cause postural instability, which increases the risk of falls and falls-related injuries [22].

The balance disorders and the risk of falls may be evaluated with numerous clinical and laboratory tests. The most common clinical balance deficits assessment tests in elderly and PD are the Berg Balance Scale (BBS), Tinneti test, Functional Reach Test (FRT) and the Timed Up and Go test (TUG) [11], [19]. These tests are subjective in nature and asses mainly the ability to maintenance balance during daily tasks. They are less suitable for postural control mechanisms evaluation.

\footnotetext{
* Corresponding author: Grzegorz Juras, Institute of Sport Sciences, The Jerzy Kukuczka Academy of Physical Education in Katowice, ul. Mikołowska 72a, 40-065 Katowice, Poland. E-mail: g.juras@awf.katowice.pl

Received: September 3rd, 2019

Accepted for publication: November 28th, 2019
} 
It is also known that in accordance with EvidenceBased Medicine policy, an objective assessment is the most valuable and desired scientific method.

The static and dynamic posturography is most suitable objective postural control mechanisms evaluation, which have experimental and clinical relevance [7], [10], [26]. It is known that some authors proposed a novel approach in assessing postural control, where they use more advanced signal analysis [13] or the combination of the static posturography with virtual environment [12]. Moreover, it is recognized that the pathophysiological mechanisms of postural control disturbances in PD might be better understood using posturography [24]. That is why posturography has become a popular objective diagnostic tool in Parkinson's disease. However, it is known that there are many different procedures in posturography where the researchers use various measurement durations, conditions, variables and equipment [14], what often results in contradictory measurement outcomes.

Postural instability is a major problem in Parkinson's disease, which may exist during quiet stance, but also during everyday tasks, such as walking, turning, crossing obstacle, standing up from sitting and gait initiation [9]. Gait initiation challenges the postural control system as it engages a voluntary transition from a stable static configuration of the body to an unsteady, dynamic configuration [29]. In the laboratory settings, gait is usually evaluated with the use of the three-dimensional motion capture optoelectronic systems. This method provides kinematic and kinetic data characterising gait. Unfortunately, this method is rarely used in clinical practice due to several restrictions such as high costs of equipment, lack of large and separate room and long-lasting complicated results analysis.

Recent studies reported that step initiation can be measured with the use of force platform though novel posturographic procedure, which evaluate gait initiation in different conditions [5], [27]. We have used this approach to assess step initiation in PD patients and healthy elderly. The aim of this study was to evaluate the reliability of the measurements calculated with the novel posturographic procedure using the intra class coefficient (ICC) method and to investigate the relationships between parameters of these procedures and clinical tests. We hypothesised that proposed gait initiation test is a valid and reliable balance and motor disability assessment, especially in patients with neurological deficits. We have also assumed high significant correlation between parameters of step initiation procedures and clinical tests.

\section{Materials and methods}

\section{Participants}

Thirty-five subjects with idiopathic PD and thirty-five aged-matched healthy control subjects participated in this study. The demographic and clinical characteristics of the subjects are presented in Table 1 . There were no significant differences between the groups for age, body mass and body height. Written informed consent was acquired from the participants before their participation in the study. PD patients were tested during „ON” period at their usual antiparkinsonian medication (at least one hour). At the time of testing, none of the patients exhibited any dyskinesia, dystonia or other signs of involuntary movement. Subjects were assessed with the following clinical test: UPDRS part III, Berg Balance Scale (BBS), Timed Up and Go test (TUG), Tinetti test, Functional Reach Test (FRT).

Inclusion criteria for PD patients were: (1) diagnosed Parkinson's disease, (2) being on antiparkinsonian medication treatment. Exclusion criteria were: (1) no consent to take part in the experiment, (2) dementia and cognitive impairment based on results of the Mini Mental Status Examination (MMSE), (3) neuromuscular, vestibular or orthopaedic disorders.

Table 1. The demographic and clinical characteristics of the subjects

\begin{tabular}{|l|c|c|}
\hline & $\begin{array}{c}\text { Parkinson's } \\
\text { disease subjects }\end{array}$ & $\begin{array}{c}\text { Elderly } \\
\text { healthy subjects }\end{array}$ \\
\hline$N$ & 35 & 35 \\
\hline age [years] & $66.2 \pm 8.3$ & $68.9 \pm 5.9$ \\
\hline body weight [kg] & $73.1 \pm 9.5$ & $69.5 \pm 9.7$ \\
\hline body height [cm] & $169.1 \pm 6.2$ & $166.3 \pm 7.3$ \\
\hline H\&Y & II - III & - \\
\hline UPDRS-III [pts] & $19.1 \pm 9.8$ & - \\
\hline BBS [pts] & $48.3 \pm 8.8$ & $55.2 \pm 1.4$ \\
\hline Tinneti [pts] & $21.9 \pm 6.2$ & $27.9 \pm 0.3$ \\
\hline FRT [cm] & $23.7 \pm 7.9$ & $29.8 \pm 5.5$ \\
\hline TUG [s] & $10.1 \pm 4.0$ & $6.4 \pm 1.0$ \\
\hline MMSE [pts] & $27.5 \pm 2.5$ & $28.2 \pm 1.8$ \\
\hline
\end{tabular}

\section{Procedure of gait initiation}

The gait initiation was measured on the two force platforms (AMTI, AccuGait) with the use of AMTI NetForce software. The platforms sampling frequency was set to $100 \mathrm{~Hz}$. The raw data were processed offline with dual-pass $7 \mathrm{~Hz}$ low-pass Butterworth filter using MATLAB software (Mathworks, Natic, MA). The procedure consisted of three phases: (1) quiet standing 
on the first platform for 15 seconds, (2) crossing on the second platform after an acoustic cue, (3) quiet standing on a second platform until measurement completion (Fig. 1). There were four different conditions: crossing without obstacle (unperturbed trial), crossing the obstacle (crossing obstacle), stepping up (step-up) and stepping down (step-down) (Fig. 2). Each trial lasted 35 seconds and was repeated three times.

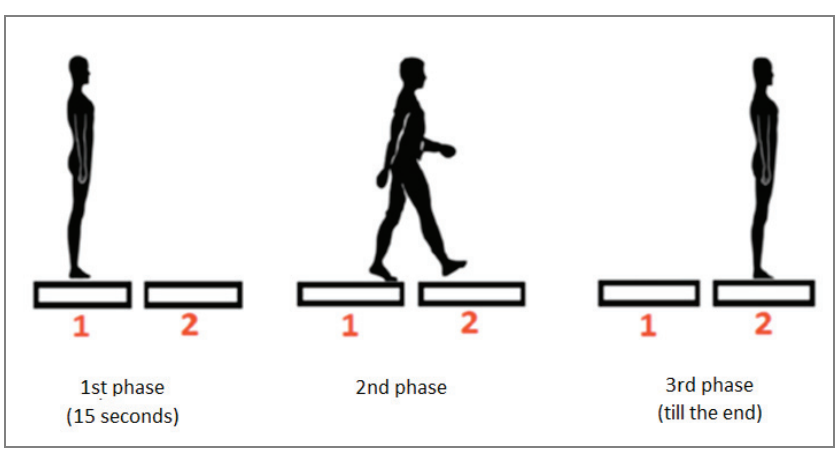

Fig. 1. The experimental set-up

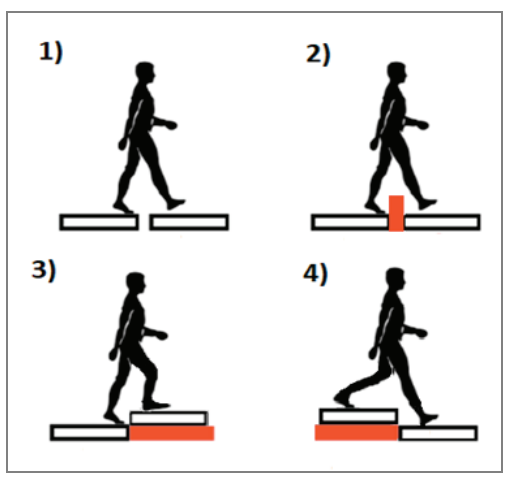

Fig. 2. Four conditions of the step initiation test

\section{Data analysis}

The center of foot pressure (COP) displacements data were collected in all three phases. The recording was divided into phases using an algorithm based on the limit of instantaneous COP displacement. The gait initiation begins when momentary COP displacement exceed the mean value of the instantaneous COP increased by three standard deviations (SD). This threshold was calculated based on measurements made within the first $5 \mathrm{~s}$ of the test (Phase 1). The step phase was finished when momentary COP displacement was lower than mean value plus three SDs based on the last $5 \mathrm{~s}$ of the trial (Phase 3).

The following variables were analysed:

1. Phase 1 and Phase 3:

- vCOP - the antero-posterior (AP) and medio-lateral (ML) COP velocity $[\mathrm{cm} / \mathrm{s}]$,
- raCOP - the antero-posterior (AP) and medio-lateral (ML) range of COP [cm],

- rmsCOP - the antero-posterior (AP) and medio-lateral (ML) root mean square of COP [cm].

2. Phase 2:

- S1 - the stability time from loss of stability to step on the second platform [s] (stability time 1),

- S2 - the stability time from step on the second platform until the stability is regained [s] (stability time 2 ),

- transit time: time from exit from stability state until gaining post-transit stability [s].

\section{Statistical analysis}

The reliability of all measurements was estimated with the use of intraclass correlation coefficients (ICCs) according to Shrout and Fleiss [23]. Derived from the ANOVA results, the ICC compares within subject variability and between subject variability. The levels of reliability were considered poor $(I C C<0.40)$, moderate $(0.40 \leq I C C<0.60)$, good $(0.60 \leq I C C<0.80)$, and excellent $(I C C>0.80)$ [16]. The non-parametric Spearman's rank test was used to established correlations between all posturographic variables and scores of standard clinical tests.

\section{Results}

\section{Reliability - PD patients}

In gait initiation procedure, for phase 1 in all conditions for both AP and ML directions, the vCOP, raCOP and rmsCOP showed an excellent reliability $(I C C>0.80)$. For phase 2 , reliability of all variables in all conditions also was excellent $(I C C=0.8-0.9)$. For phase 3 , in unperturbed trial the vCOP, raCOP and rmsCOP showed somewhat lower, but yet good, reliability $(I C C=0.7-0.9)$. In other conditions all variables presented an excellent reliability $(I C C>0.8)$. In Table 2, the reliability results are summarized.

\section{Reliability - elderly healthy subjects}

ICC scores for phase 1 in all conditions in AP direction were good to excellent $(I C C=0.6-0.9)$, but for ML direction in obstacle crossing, the reliability of raCOP and rmsCOP was moderate $(I C C=0.4)$. However, in other conditions, all variables $I C C$ values were excellent $(I C C=0.8-0.9)$. For phase 2, reliability of all variables in all conditions was good and excellent $(I C C=0.7-0.9)$. For phase 3 in unperturbed and obstacle trials, the raCOP, rmsCOP 
Table 2. ICC values in 30-s trial, repeated three times

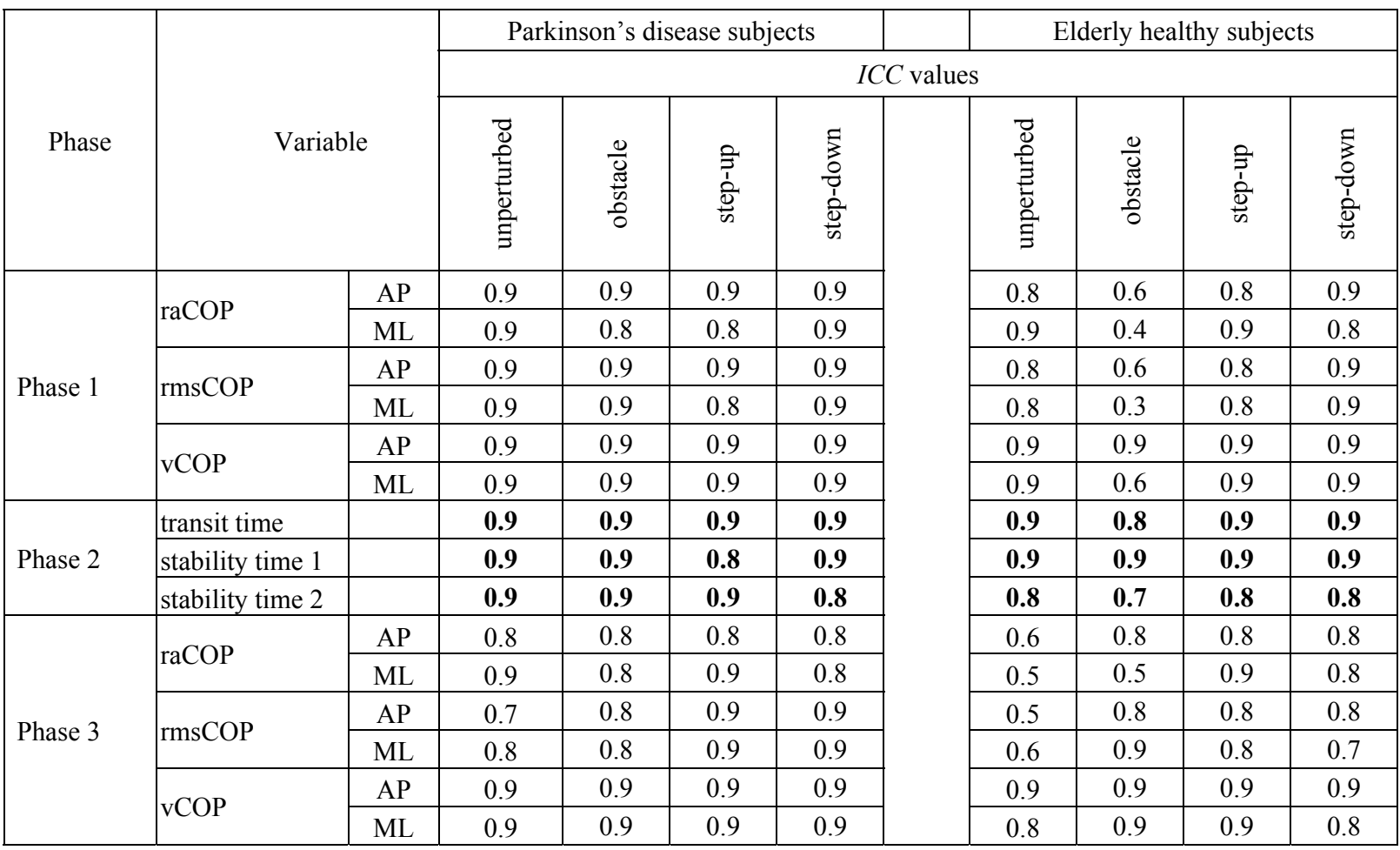

and $\mathrm{VCOP}$ in AP direction presented a good and excellent reliability $(I C C=0.6-0.9)$, in $\mathrm{ML}$ direction the $\mathrm{vCOP}$, raCOP and rmsCOP showed somewhat lower ICC values $(I C C=0.5-0.9)$. However, in other conditions, all variables ICC values were good and excellent $(I C C=0.7-0.9)$ (Table 2$)$.

\section{Relationship between posturographic variables and clinical tests}

The results of correlation analysis are listed in Tables 3 and 4 for PD patients and controls, respectively. While only few associations were found be- tween Tinetti scores and posturographic variables in controls, in PD patients the significant correlations were found between the posturography measures and the UPDRS, Tinetti, FRT, TUG and with the BBS. There was a positive correlation between the range, velocity and root mean square of COP in both phases 1 and 3 and disease severity evaluated with UPDRS in all trial conditions. Also, there was a positive relationship between transit time, stability time 1, stability time 2 and UPDRS. Furthermore, there were inverse relationships between the static and dynamic posturography measures and BBS, Tinetti test, FRT and with the TUG (Table 3).

Table 3. Results of correlation analysis for patients with Parkinson's Disease

\begin{tabular}{|c|c|c|c|c|c|c|c|c|c|c|c|c|}
\hline \multirow{2}{*}{ Phase } & & & UPDRS & BBS & FRT & Tinetti & TUG & UPDRS & BBS & FRT & Tinetti & TUG \\
\hline & & & \multicolumn{5}{|c|}{ unperturbed } & \multicolumn{5}{|c|}{ obstacle } \\
\hline \multirow{6}{*}{ Phase 1} & \multirow{2}{*}{$\mathrm{raCOP}$} & AP & 0.51 & -0.31 & -0.21 & -0.30 & 0.17 & 0.39 & -0.35 & -0.14 & -0.34 & 0.19 \\
\hline & & ML & 0.39 & -0.13 & -0.11 & -0.25 & 0.07 & 0.33 & -0.12 & -0.06 & -0.19 & 0.09 \\
\hline & \multirow{2}{*}{$\mathrm{rmsCOP}$} & $\mathrm{AP}$ & 0.47 & -0.31 & -0.21 & -0.30 & 0.20 & 0.37 & -0.26 & -0.08 & -0.23 & 0.15 \\
\hline & & ML & 0.35 & -0.14 & -0.13 & -0.27 & 0.09 & 0.32 & -0.09 & -0.03 & -0.15 & 0.02 \\
\hline & \multirow{2}{*}{ vCOP } & $\mathrm{AP}$ & 0.53 & -0.38 & -0.46 & -0.34 & 0.21 & 0.49 & -0.30 & -0.36 & -0.29 & 0.13 \\
\hline & & ML & 0.35 & -0.15 & -0.14 & -0.22 & 0.07 & 0.29 & -0.10 & -0.08 & -0.17 & 0.03 \\
\hline \multirow{3}{*}{ Phase 2} & \multicolumn{2}{|l|}{ transit time } & $\mathbf{0 , 5 8}$ & -0.39 & -0.31 & -0.57 & 0.19 & 0.58 & -0.35 & -0.38 & -0.42 & 0.27 \\
\hline & \multicolumn{2}{|l|}{ time S1 } & 0,66 & -0.52 & -0.51 & -0.66 & 0.39 & 0.67 & -0.44 & -0.42 & -0.54 & 0.37 \\
\hline & \multicolumn{2}{|l|}{ time S2 } & 0.42 & -0.30 & -0.27 & -0.46 & 0.17 & 0.52 & -0.30 & -0.44 & -0.38 & 0.21 \\
\hline
\end{tabular}


Table 3 continued

\begin{tabular}{|c|c|c|c|c|c|c|c|}
\hline \multirow{6}{*}{ Phase 3} & \multirow{2}{*}{$\mathrm{raCOP}$} & AP & 0.34 & -0.41 & -0.21 & -0.51 & 0.34 \\
\hline & & ML & 0.33 & -0.27 & -0.17 & -0.41 & 0.27 \\
\hline & \multirow{2}{*}{$\mathrm{rmsCOP}$} & $\mathrm{AP}$ & 0.28 & -0.36 & -0.14 & -0.43 & 0.28 \\
\hline & & ML & 0.35 & -0.18 & -0.13 & -0.37 & 0.12 \\
\hline & \multirow{2}{*}{ vCOP } & $\mathrm{AP}$ & 0.56 & -0.44 & -0.41 & -0.52 & 0.39 \\
\hline & & ML & 0.33 & -0.22 & -0.14 & -0.44 & 0.24 \\
\hline & & & \multicolumn{5}{|c|}{ step-up } \\
\hline \multirow{6}{*}{ Phase 1} & \multirow{2}{*}{$\mathrm{raCOP}$} & AP & 0.33 & -0.20 & -0.11 & -0.26 & 0.13 \\
\hline & & ML & 0.27 & -0.20 & -0.14 & -0.38 & 0.20 \\
\hline & \multirow{2}{*}{$\mathrm{rmsCOP}$} & $\mathrm{AP}$ & 0.29 & -0.17 & -0.07 & -0.14 & 0.07 \\
\hline & & ML & 0.34 & -0.19 & -0.15 & -0.29 & 0.12 \\
\hline & \multirow{2}{*}{ vCOP } & $\mathrm{AP}$ & 0.40 & -0.24 & -0.39 & -0.28 & 0.09 \\
\hline & & ML & 0.29 & -0.03 & -0.10 & -0.18 & -0.01 \\
\hline \multirow{3}{*}{ Phase 2} & \multicolumn{2}{|l|}{ transit time } & 0.62 & -0.39 & -0.35 & -0.38 & 0.20 \\
\hline & \multicolumn{2}{|l|}{ time S1 } & 0.67 & -0.43 & -0.36 & -0.46 & 0.19 \\
\hline & \multicolumn{2}{|l|}{ time S2 } & 0.51 & -0.32 & -0.35 & -0.33 & 0.12 \\
\hline \multirow{6}{*}{ Phase 3} & \multirow{2}{*}{$\mathrm{raCOP}$} & $\mathrm{AP}$ & 0.17 & -0.29 & -0.14 & -0.43 & 0.29 \\
\hline & & ML & 0.20 & -0.24 & -0.13 & -0.47 & 0.29 \\
\hline & \multirow{2}{*}{$\mathrm{rmsCOP}$} & $\mathrm{AP}$ & 0.24 & -0.34 & -0.14 & -0.41 & 0.30 \\
\hline & & ML & 0.28 & -0.25 & -0.14 & -0.51 & 0.27 \\
\hline & \multirow{2}{*}{ vCOP } & $\mathrm{AP}$ & 0.41 & -0.39 & -0.43 & -0.50 & 0.44 \\
\hline & & ML & 0.33 & -0.34 & -0.25 & -0.54 & 0.36 \\
\hline
\end{tabular}

\begin{tabular}{|l|c|c|c|c|}
\hline $\mathbf{0 . 3 4}$ & -0.33 & -0.08 & $\mathbf{- 0 . 5 0}$ & 0.23 \\
\hline 0.25 & -0.26 & -0.04 & $\mathbf{- 0 . 5 0}$ & 0.29 \\
\hline 0.32 & -0.30 & -0.10 & $-\mathbf{0 . 5 0}$ & 0.16 \\
\hline $\mathbf{0 . 3 8}$ & -0.31 & -0.10 & $\mathbf{- 0 . 5 0}$ & 0.32 \\
\hline $\mathbf{0 . 5 0}$ & $\mathbf{- 0 . 3 9}$ & -0.32 & $\mathbf{- 0 . 5 1}$ & 0.28 \\
\hline $\mathbf{0 . 3 6}$ & -0.33 & -0.21 & $\mathbf{- 0 . 5 6}$ & $\mathbf{0 . 3 3}$ \\
\hline \multicolumn{5}{|c|}{ step-down } \\
\hline 0.30 & -0.19 & -0.25 & -0.24 & 0.09 \\
\hline 0.15 & -0.08 & -0.23 & -0.07 & 0.05 \\
\hline 0.19 & -0.10 & -0.15 & -0.14 & 0.03 \\
\hline 0.09 & 0.06 & -0.08 & 0.06 & -0.12 \\
\hline $\mathbf{0 . 3 7}$ & -0.30 & $-\mathbf{0 . 3 4}$ & -0.22 & 0.12 \\
\hline 0.21 & -0.10 & -0.21 & -0.07 & -0.02 \\
\hline $\mathbf{0 . 5 9}$ & $\mathbf{- 0 . 4 2}$ & $\mathbf{- 0 . 5 4}$ & $\mathbf{- 0 . 4 5}$ & 0.33 \\
\hline $\mathbf{0 . 6 4}$ & $\mathbf{- 0 . 4 0}$ & $-\mathbf{0 . 5 6}$ & $-\mathbf{0 . 5 1}$ & 0.33 \\
\hline $\mathbf{0 . 4 4}$ & -0.35 & $-\mathbf{0 . 4 0}$ & $\mathbf{- 0 . 3 9}$ & 0.24 \\
\hline 0.28 & $\mathbf{- 0 . 3 4}$ & -0.11 & -0.33 & 0.23 \\
\hline $\mathbf{0 . 4 0}$ & -0.19 & -0.11 & -0.33 & 0.19 \\
\hline 0.32 & $\mathbf{- 0 . 3 6}$ & -0.13 & $\mathbf{- 0 . 4 2}$ & 0.26 \\
\hline $\mathbf{0 . 4 4}$ & -0.16 & -0.13 & -0.33 & 0.15 \\
\hline $\mathbf{0 . 5 4}$ & $\mathbf{- 0 . 3 9}$ & $\mathbf{- 0 . 4 4}$ & $\mathbf{- 0 . 4 1}$ & 0.25 \\
\hline $\mathbf{0 . 4 3}$ & -0.28 & -0.20 & $\mathbf{- 0 . 3 6}$ & 0.23 \\
\hline
\end{tabular}

Table 4. Results of correlation analysis for elderly healthy subjects

\begin{tabular}{|c|c|c|c|c|c|c|c|c|c|c|}
\hline Phase & & & BBS & FRT & Tinetti & TUG & BBS & FRT & Tinetti & TUG \\
\hline & & & \multicolumn{4}{|c|}{ unperturbed } & \multicolumn{4}{|c|}{ obstacle } \\
\hline \multirow{6}{*}{ Phase 1} & \multirow{2}{*}{$\mathrm{raCOP}$} & AP & -0.03 & -0.03 & -0.10 & 0.08 & -0.03 & -0.03 & -0.10 & 0.08 \\
\hline & & ML & 0.07 & 0.18 & 0.00 & -0.04 & 0.07 & 0.18 & 0.00 & -0.04 \\
\hline & \multirow{2}{*}{$\mathrm{rmsCOP}$} & AP & -0.08 & -0.09 & -0.17 & 0.13 & -0.08 & -0.09 & -0.17 & 0.13 \\
\hline & & ML & 0.02 & 0.09 & -0.06 & 0.02 & 0.02 & 0.09 & -0.06 & 0.02 \\
\hline & \multirow{2}{*}{ vCOP } & $\mathrm{AP}$ & -0.23 & 0.02 & -0.24 & 0.28 & -0.23 & 0.02 & -0.24 & 0.28 \\
\hline & & ML & -0.08 & 0.05 & -0.07 & 0.10 & -0.08 & 0.05 & -0.07 & 0.10 \\
\hline \multirow{3}{*}{ Phase 2} & \multicolumn{2}{|c|}{ transit time } & 0.31 & 0.02 & 0.33 & 0.05 & 0.31 & 0.02 & 0.33 & 0.05 \\
\hline & \multicolumn{2}{|l|}{ time S1 } & 0.18 & -0.13 & 0.38 & 0.21 & 0.18 & -0.13 & 0.38 & 0.21 \\
\hline & \multicolumn{2}{|l|}{ time S2 } & 0.22 & -0.08 & 0.14 & 0.08 & 0.22 & -0.08 & 0.14 & 0.08 \\
\hline \multirow{7}{*}{ Phase 3} & \multirow{2}{*}{ raCOP } & $\mathrm{AP}$ & -0.25 & -0.12 & -0.18 & -0.02 & -0.25 & -0.12 & -0.18 & -0.02 \\
\hline & & ML & -0.03 & -0.16 & 0.11 & -0.17 & -0.03 & -0.16 & 0.11 & -0.17 \\
\hline & \multirow{2}{*}{$\mathrm{rmsCOP}$} & AP & -0.15 & -0.29 & -0.20 & 0.01 & -0.15 & -0.29 & -0.20 & 0.01 \\
\hline & & ML & 0.03 & -0.25 & 0.11 & -0.15 & 0.03 & -0.25 & 0.11 & -0.15 \\
\hline & \multirow{2}{*}{ vCOP } & $\mathrm{AP}$ & -0.29 & -0.03 & -0.42 & 0.24 & -0.29 & -0.03 & -0.42 & 0.24 \\
\hline & & ML & -0.20 & -0.11 & 0.04 & -0.02 & -0.20 & -0.11 & 0.04 & -0.02 \\
\hline & & & \multicolumn{4}{|c|}{ step-up } & \multicolumn{4}{|c|}{ step-down } \\
\hline \multirow{6}{*}{ Phase 1} & \multirow{2}{*}{ raCOP } & $\mathrm{AP}$ & -0.30 & 0.01 & -0.52 & 0.07 & -0.17 & 0.04 & -0.11 & 0.03 \\
\hline & & ML & -0.13 & -0.04 & -0.18 & 0.09 & -0.25 & -0.22 & -0.29 & -0.11 \\
\hline & \multirow{2}{*}{ rmsCOP } & AP & -0.30 & -0.11 & -0.50 & 0.13 & -0.17 & 0.05 & -0.12 & -0.04 \\
\hline & & ML & -0.20 & -0.12 & -0.21 & 0.12 & -0.21 & -0.24 & -0.21 & -0.10 \\
\hline & \multirow{2}{*}{ vCOP } & AP & -0.43 & -0.05 & -0.54 & 0.40 & -0.24 & -0.03 & -0.34 & 0.28 \\
\hline & & ML & -0.24 & 0.07 & -0.28 & 0.09 & -0.11 & -0.06 & -0.09 & -0.05 \\
\hline \multirow{3}{*}{ Phase 2} & \multicolumn{2}{|l|}{ transit time } & 0,16 & 0.06 & -0.01 & -0.04 & -0.15 & -0.01 & -0.24 & -0.09 \\
\hline & \multicolumn{2}{|l|}{ time S1 } & 0,27 & 0.24 & 0.19 & -0.23 & -0.13 & -0.02 & -0.27 & -0.10 \\
\hline & \multicolumn{2}{|l|}{ time S2 } & 0,19 & -0.03 & 0.02 & 0.06 & 0.00 & -0.04 & -0.04 & 0.04 \\
\hline
\end{tabular}


Table 4 continued

\begin{tabular}{|c|c|c|c|c|c|c|c|c|c|c|}
\hline \multirow{6}{*}{ Phase 3} & \multirow{2}{*}{$\mathrm{raCOP}$} & AP & -0.32 & -0.19 & -0.14 & 0.11 & -0.32 & -0.02 & -0.23 & -0.15 \\
\hline & & ML & -0.12 & -0.01 & -0.12 & -0.10 & 0.05 & 0.04 & -0.03 & -0.25 \\
\hline & \multirow{2}{*}{$\mathrm{rmsCOP}$} & AP & -0.33 & -0.28 & -0.18 & 0.13 & -0.27 & -0.02 & -0.19 & -0.10 \\
\hline & & ML & -0.05 & -0.02 & -0.07 & -0.03 & -0.20 & -0.08 & -0.09 & -0.08 \\
\hline & \multirow{2}{*}{ vCOP } & $\mathrm{AP}$ & -0.29 & 0.08 & -0.28 & 0.21 & -0.53 & -0.09 & -0.46 & 0.26 \\
\hline & & ML & -0.23 & -0.05 & -0.11 & 0.07 & -0.18 & -0.01 & -0.03 & 0.03 \\
\hline
\end{tabular}

\section{Discussion}

The main objective of this study was to assess the intra-rater reliability of the measurements obtained with the proposed procedure of gait initiation in PD patients and the elderly. The method allowed for the assessment of various aspects of dynamic balance during gait initiation in different conditions. Thanks to its usage, one can gain new important information about body stability before and after completing motor task and stability during making a step.

In clinical practice balance impairments are evaluated by numerous standard functional tests, which are also reliable methods. BBS has been the main tool used to assess balance deficits in different disorders; however, this scale was originally designed to evaluate the risk of falling in elderly. The reliability of the questionnaire is excellent $(I C C=0.6$ to 0.9 for all items) [1]. Similarly, the Tinetti test reaches good to excellent reliability $(I C C>0.8)[15]$ and the results of TUG present excellent reliability $(I C C=0.9)$ [20]. However, as mentioned before, all these tests are subjective, highly dependent on the skills and experiences of the assessor.

Traditional objective gait analysis is conducted with three-dimensional motion analysis systems, which has been approved as a valuable tool in the assessment of gait disorders and objective evaluation of therapy. Reliability of kinematic data from these systems is high for motion in the sagittal plane $(I C C=0.97-0.99)$, the frontal plane $(I C C=0.76-0.98)$, and the transverse plane $(I C C=0.73-0.90)$ [28]. However, this method assesses different aspects of gait and step initiation compared to procedure proposed in this study. The proposed procedure of dynamic balance assessment during gait initiation is an objective method, as reliable as three-dimensional motion analysis systems, moreover, it is less expensive and simpler. The most valuable measurements of the novel procedure are stability time 1 , stability time 2 and transit time. From the results of the present study, these parameters demonstrate excellent reliability in Parkinson's disease patients $(I C C=$ $0.8-0.9)$ and the elderly $(I C C=0.7-0.9)$.
Parkinson's disease patients present balance and gait deficits and step initiation problems. The gait initiation problems in PD are associated with bradykinesia and the prolonged preparatory time for movement, what may reflect motor programming issues [17], [18]. The presented procedure easily assess these impairments of gait initiation, the stability time 1 and the transit time may evaluate the signs of bradykinesia and impairments of preparatory phase of gait. Also, it is a reliable tool to detect the problems with regaining stability after making a step (stability time 2), what may reflect impaired active control of antigravity muscles before the foot-contact of the swing limb (active braking) [4]. Moreover, it is well known that PD patients present increased postural instability and gait disorders during performing an additional task [6], [21], therefore a complex motor task such as crossing obstacle or walking stairs maight be demanding in PD. Thus, our novel procedure evaluate gait initiation in different condition, which are typical for daily living, what may be helpful in an objective diagnosis of the risk of falling in PD during their everyday tasks.

Furthermore, the stability time 1, stability time 2 and transit time values in PD were positively correlated with UPDRS motor examination scores, TUG and negatively correlated with BBS, Tinetti test and FRT scores, what indicate the close association of static and dynamic balance to gait. However, only a few correlation between posturographic measurements and clinical tests scores in control group were recorded, what may testify that used tests are dedicated to patients with motor disorders.

In recent study on PD patients, Barbosa et al. [2] found a weak correlation between static posturography and BBS $(r=-0.34, p<0.05)$. Similar results were obtained by other authors [8], who recorded a significant negative correlation between BBS and SD COP in the frontal plane $(r=-0.49, p=0.007)$ and the area of $\operatorname{COP}(r=-0.53,=0.004)$. Moreover, Souza et al. [26] showed no significant correlations between BBS, TUG and static posturography, but presented significant correlations with dynamic balance test. The authors reported a high correlation between time 
to perform TUG and the duration of obstacle crossing, what is in accordance with the results of our study, e.g., the significant positive correlation between TUG and the stability time 1 during both crossing without obstacle $(r=0.39, p<0.05)$, and obstacle crossing $(r=0.37, p<0.05)$. Moreover, the stability time 1 is the variable that highly correlate with clinical tests in all conditions, especially during obstacle crossing, walking up and down the step. Each clinical test and motor task requires postural adjustment, motor planning and correct movement sequence [26]. Our results indicate that the longer the preparatory time for the movement, the longer the time of TUG performance. Also, the longer the stability time 1, the less points patients gained in BBS, Tinetti test or FRT, which is associated with increased risk of falling and impaired stability. Similar relationships can be noted in transit time, which also shows numerous negative correlations with the FRT, Tinetti and BBS. Therefore, on the basis of these results, it can be concluded that the transit time and stability time 1 are good predictors of disorders during preparatory time of gait initiation.

There are some limitations to the use of the proposed procedure for assessing patients with PD. It has limited usefulness for evaluating the dynamic balance of individuals in the later stages of the disease stage IV or stage $\mathrm{V}$ of the $\mathrm{H}$ and $\mathrm{Y}$ scale. Furthermore, the standard gait evaluation is based on COM-COP analysis, and the proposed procedure is, in our opinion, a simplified assessment. Nevertheless, it is still useful in clinical practice.

Results of this investigation indicate that, among several parameters of the gait initiation test, the most valuable are the stability time 1 , stability time 2 and transit time, when administered to PD patients and healthy adults. Using three repetitions of trial allowed for reliable measurement of dynamic balance. Based on the results of this study, it can be stated that a newly developed gait initiation procedure is reliable and very suitable for the assessment of patients with Parkinson disease, and that it can be used for an objective assessment of the clinical condition and dynamic balance, what may be usable during programming appropriate rehabilitation and treatment.

\section{Acknowledgements}

The study was supported by the National Center for Research and Development Grant under the program STRATEG MED III within the "VB-Clinic" project No. STRATEGMED3/306011/ $1 / \mathrm{NCBR} / 2017$. The authors declare no conflicts of interest.

\section{References}

[1] Babaei-Ghazani A., Hosein M., Shahidi G.A., Habibi S.A.H., Forogh B., Ahadi T., Eftekharsadat B., Reliability and validity of the Persian translation of Berg Balance Scale in Parkinson's disease, Aging. Clin. Exp. Res., 2016, 1-6.

[2] Barbosa A.F., de Oliveira Souza C., Chen J., Francato D.V., Caromano F.A., Chien H.F., Barbosa E.R., D'AndreA GREVE J.M., Voos M.C., The competition with a concurrent cognitive task affects posturographic measures in patients with Parkinson's Disease, Arq. Neuro-Psiquiat., 2015, 73 (11), 906-912.

[3] BŁaszczyk J.W., Czerwosz L., Postural stability in the process of aging, Gerontol. Pol., 2005, 13 (1). 25-36.

[4] Chastan N., Do M. C., Bonneville F., Torny F., Bloch F., Westby G.W.M., Dormont D., AgID Y., Welter M.L., Gait and balance disorders in Parkinson's Disease: Impaired active braking of the fall of centre of gravity, Mov. Disord., 2009, 24 (2), 188-195.

[5] Chmielewska D., Sobota, G., Stania M., BŁaszczak E., SŁOMKA K.J., JuRAS G., A comparison of a step-initiation task in women with and without urinary incontinence. A casecontrol study, Neurourol. Urodynam., 2018, 1-7.

[6] Doan J.B., De Bruin N., Pellis S.M., Suchowersky O., Whishaw I.Q., BROWN L.A., Obstacle avoidance amongst Parkinson's Disease patients is challenged in a threatening context, J. Neurodegener. Dis., 2013, 1-8.

[7] Ebersbach G., Gunkel M., Posturography reflects clinical imbalance in Parkinson's Disease, Mov. Disord., 2011, 26 (2), 241-246.

[8] Ferrazzoli D., Fasano A., Maestri R., Bera R., Palamara G., Ghilardi M.F., Pezzoli G., Frazzitta G., Balance dysfunction in Parkinson's Disease. The role of posturography in developing a rehabilitation program, Parkinson's Disease, 2015, 1-10.

[9] Hass C.J., Waddell D.E., Fleming R.P., Juncos J.L., GregOR R.J., Gait initiation and dynamic balance control in Parkinson's Disease, Arch. Phys. Med. Rehab., 2005, 86, 2172-2176.

[10] Ickenstein G.W., Аmbach H., Klöditz A., КосH H., ISENMANN S., REICHMANN H., ZIEMSSEN T., Static posturography in aging and Parkinson's Disease, Front Aging. Neurosci., 2012, 4, 1-7.

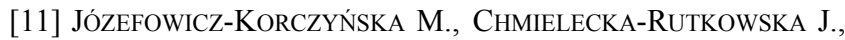
Mazerant A., Clinical tests for balance stability and gait assessment - Bedside Tests, Pol. Prz. Otorynolaryngol., 2016, $5,57-63$

[12] JuRkojć J., Wodarski P., Bieniek A., Gzik M., Michnik R., Influence of changing frequency and various sceneries on stabilometric parameters and on the effect of adaptation in an immersive $3 D$ virtual environment, Acta Bioeng. Biomech., 2017, 19 (3), 129-137

[13] JuRKoJĆ J., Balance disturbances coefficient as a new value to assess ability to maintain balance on the basis of FFT curves, Acta Bioeng. Biomech., 2018, 20 (1), 143-151.

[14] Kamieniarz A., Michalska J., Brachman A., PawŁowski M., SŁOMKA K.J., JuRAS G., A posturographic procedure assessing balance disorders in Parkinson's disease: a systematic review, Clin. Interv. Aging., 2018, 12 (13), 2301-2316.

[15] Kegelmeyer D.A, Kloos A.D., Thomas K.M., KostyK S.K., Research report reliability and validity of the Tinetti Parkinson's Disease, Phys. Ther., 2007, 87, 1369-1378. 
[16] Mancini M., Carlson-Kuhta P., Zampieri C., Nutt, J.G., CHIARI L., HoRAK F.B., Postural sway as a marker of progression in Parkinson's disease: A pilot longitudinal study, Gait Posture., 2012, 36, 471-476.

[17] Martin M.,, Shinberg M., Kuchibhatla M., Ray L., CAROLLO J.J., SCHENKMAN M.L., Gait initiation in community-dwelling adults with Parkinson's Disease: Comparison with older and younger adults without the Disease, Phys. Ther., 2002, 82 (6), 566-577.

[18] Morris M.E., HuXham F., McGinley J., Dodd K., IANSEK R., The biomechanics and motor control of gait in Parkinson's Disease, Clin. Biomech., 2001, 16 (6), 459-470.

[19] Opara J., MaŁecki A., MaŁecka E., Socha T., Motor assessment in Parkinson's Disease, Ann. Agr. Env. Med., 2017, 24 (3), 411-415.

[20] Podsiadlo D., Richardson S., The timed "Up \& Go": a test of basic functional mobility for frail elderly persons, J. Am. Geriatr. Soc., 1991, 39 (2), 142-148.

[21] Rochester L., Hetherington V., Jones D., Nieuwboer A., Willems A., Kwakkel G., Van Wegen E. et al., Attending to the task: Interference effects of functional tasks on walking in Parkinson's Disease and the roles of cognition, depression, fatigue and balance, Arch. Phys. Med. Rehabil., 2004, 85, $1578-1585$.

[22] Schoneburg B., Mancini M., Horak F.B., Nutt J.G., Framework for understanding balance dysfunction in Parkinson's Disease, Mov. Disord., 2013, 28(11), 1474-1482.

[23] Shrout P.E., FLeiss J.L., Intraclass correlation: uses in assessing rater reliability, Psychol. Bull., 1979, 86, 420-428.
[24] Snijders A.H., Leunissen I., BAKker M., Overeem S., Helmich R.C., Bloem B.R., Toni I., Gait-related cerebral alterations in patients with Parkinson's Disease with freezing of gait, Brain, 2011, 134(1), 59-72.

[25] Soto-VARela A., Rossi-IzQuierdo M., Faraldo-García A., VAAMONDE-SÁNCHEZ-ANDRADE I., GAYOSO-Diz P., LIROLA-Delgado A., SAntos-PÉrez S., Balance disorders in the elderly: Does instability increase over time?, Ann. Oto. Rhino. Laryn., 2016, 125(7), 550-558.

[26] Souza C., Voos M.C., Barbosa A.F., Chen J., Francato D.C.V., Milosevic M., Popovic M., Fonoff E.T., Chien H.F., BARBOSA E.R., Relationship between posturography, clinical balance and executive function in Parkinson's Disease, J. Motor. Behav., 2018, 1-10.

[27] Stania M., Sarat-Spek A., Blacha T., KazeK B., SŁomka K.J., Emich-Widera E., JuRAs G., Step-Initiation Deficits in Children with Faulty Posture Diagnosed with Neurodevelopmental Disorders during Infancy, Front Pediatr., 2017, 5 (239), 1-7.

[28] Tsushima H., Morris M.E., McGinley J., Test-Retest Reliability and Inter-Tester Reliability of Kinematic Data from a Three-Dimensional Gait Analysis System, J. Jpn. Phys. Ther. Assoc., 2003, 6, 9-17.

[29] Vallabhajosula S., Buckley T.A., Tillman M.D., Hass C.J., Age and Parkinson's disease related kinematic alterations during multi-directional gait initiation, Gait Posture, 2013, 37, 280-286.

[30] Whipple R., Wolfson L., Derby C., Singh D., Tobin J., Altered sensory function and balance in older persons, J. Gerontol., 1993, 48 (8), 71-76.

[31] WinTER D.A., Human blance and posture control during standing and walking, Gait Posture, 1995, 3 (4), 193-214. 